\title{
ON THE AUTOMORPHISM GROUP OF CERTAIN SIMPLE $C^{*}$-ALGEBRAS
}

\author{
JESPER MYGIND
}

\begin{abstract}
We show that the information contained in $K L(A, B)$ is determined by other invariants when $A$ and $B$ are certain simple unital projectionless $C^{*}$-algebras. This allows us to compute the group of automorphisms modulo the group of approximately inner automorphisms in terms of the Elliott invariant.
\end{abstract}

\section{Introduction}

For a unital $C^{*}$-algebra $A$ the Elliott invariant $\mathscr{E}_{A}$ consists of the ordered group $K_{0}(A)$ with order unit, the group $K_{1}(A)$, the compact convex set $T(A)$ of tracial states, and the restriction map $r_{A}: T(A) \rightarrow S K_{0}(A)$, where $S K_{0}(A)$ denotes the state space of $K_{0}(A)$. In [3] it was proved that the Elliott invariant is a classifying invariant for the class of unital simple infinite dimensional inductive limits of sequences of finite direct sums of building blocks. A building block is a $C^{*}$-algebra of the form

$$
A\left(n, d_{1}, d_{2}, \ldots, d_{N}\right)=\left\{f \in C(\mathrm{~T}) \otimes M_{n}: f\left(x_{i}\right) \in M_{d_{i}}, i=1,2, \ldots, N\right\},
$$

where $x_{1}, x_{2}, \ldots, x_{N}$ are (different) points in $\mathrm{T}$, and $d_{1}, d_{2}, \ldots, d_{N}$ are integers dividing $n$. The points $x_{1}, x_{2}, \ldots, x_{N}$ will be called the exceptional points of $A$. By allowing $d_{i}=n$ we may always assume that $N \geq 2$.

The following calculation of the group of automorphisms modulo the group of approximately inner automorphisms is the main result of this paper.

THEOREM 1.1. Let A be a simple unital inductive limit of a sequence of finite direct sums of building blocks.

(i) If $K_{0}(A)$ is non-cyclic then $\operatorname{Aut}(A) / \overline{\operatorname{Inn}(A)}$ is isomorphic to the semidirect product

$\left(\operatorname{Hom}\left(K_{1}(A), \operatorname{Aff} T(A) / \overline{\rho_{A}\left(K_{0}(A)\right)}\right) \times \operatorname{ext}\left(K_{1}(A), K_{0}(A)\right)\right) \rtimes \operatorname{Aut}\left(\mathscr{E}_{A}\right)$, 
where the action of $\left(\varphi_{0}, \varphi_{1}, \varphi_{T}\right) \in \operatorname{Aut}\left(\mathscr{E}_{A}\right)$ is given by

$$
(\eta, e) \mapsto\left({\widetilde{\varphi_{T}}}^{-1} \circ \eta \circ \varphi_{1}^{-1}, \varphi_{0 *} \circ \varphi_{1}^{-1 *}(e)\right)
$$

for $\eta \in \operatorname{Hom}\left(K_{1}(A)\right.$, Aff $\left.T(A) / \overline{\rho_{A}\left(K_{0}(A)\right)}\right), e \in \operatorname{ext}\left(K_{1}(A), K_{0}(A)\right)$.

(ii) If $K_{0}(A) \cong \mathrm{Z}$ then $\operatorname{Aut}(A) / \overline{\operatorname{Inn}(A)}$ is isomorphic to the semi-direct product

$$
\operatorname{Hom}\left(K_{1}(A), \operatorname{Aff} T(A) / \overline{\rho_{A}\left(K_{0}(A)\right)}\right) \rtimes \operatorname{Aut}\left(\mathscr{E}_{A}\right),
$$

where the action of $\left(\varphi_{0}, \varphi_{1}, \varphi_{T}\right) \in \operatorname{Aut}\left(\mathscr{E}_{A}\right)$ is given by

$$
\eta \mapsto{\widetilde{\varphi_{T}}}^{-1} \circ \eta \circ \varphi_{1}^{-1}, \quad \eta \in \operatorname{Hom}\left(K_{1}(A), \operatorname{Aff} T(A) / \overline{\rho_{A}\left(K_{0}(A)\right)}\right) .
$$

Here $\operatorname{Aut}\left(\mathscr{E}_{A}\right)$ denotes the group of automorphisms of $\mathscr{E}_{A}$, i.e. the group of triples $\left(\varphi_{0}, \varphi_{1}, \varphi_{T}\right)$ where $\varphi_{0}$ is an automorphism of the ordered group $K_{0}(A)$ with order unit, $\varphi_{1}$ is an automorphism of $K_{1}(A)$, and $\varphi_{T}$ is an affine homeomorphism of $T(A)$ such that

$$
r_{A} \circ \varphi_{T}^{-1}(\omega)=r_{A}(\omega) \circ \varphi_{0} \quad \text { on } \quad K_{0}(A)
$$

for every $\omega \in T(A)$. Aff $T(A)$ denotes the continuous real-valued affine functions on $T(A)$ and $\rho_{A}: K_{0}(A) \rightarrow$ Aff $T(A)$ is the group homomorphism $\rho_{A}(x)(\omega)=r_{A}(\omega)(x), x \in K_{0}(A), \omega \in T(A)$.

It follows from [3, Theorem 12.2] that the algebras considered under (i) are exactly the class considered by Thomsen in [9]. Therefore part (i) of the above theorem follows from Thomsen's calculation, [9, Theorem 8.4]. Note that the term $\operatorname{ext}\left(K_{1}(A), K_{0}(A)\right)$ is not present in case (ii). This is not because it is zero. As we shall demonstrate, it is the existence of a natural map

$$
K L(A, B)_{e} \rightarrow \operatorname{Hom}(\operatorname{Tor}(U(A) / \overline{D U(A)}), \operatorname{Tor}(U(B) / \overline{D U(B)})),
$$

when $A$ and $B$ are simple unital inductive limits of finite direct sums of building blocks with $K_{0}(A) \cong K_{0}(B) \cong \mathrm{Z}$, which is responsible for this. $K L(A, B)_{e}$ denotes the subset of elements $\kappa$ in the group $K L(A, B)$ defined by Rørdam in [5] for which the induced map $\kappa_{*}: K_{0}(A) \rightarrow K_{0}(B)$ preserves the order unit, and $U(A) / \overline{D U(A)}$ is the group of unitaries $U(A)$ in $A$ modulo the closure of the commutator subgroup $D U(A)$. The map above was also crucial in the proof of the classification result in [3].

It is an interesting question whether a similar map exists in greater generality - including the case where $A$ and $B$ are arbitrary inductive limits of subhomogeneous $C^{*}$-algebras. Such a map would probably be useful in all efforts 
of classifying larger classes of simple $C^{*}$-algebras, and our main result suggests that it could influence the structure of the automorphism group as well.

It should be noted that the class of $C^{*}$-algebras considered under (ii) is quite large. It consists of matrix algebras over simple unital projectionless $C^{*}$-algebras, see [3, Corollary 12.5].

\section{Preliminaries}

The purpose of this section is to introduce the notation used in this paper and to list some results on building blocks from [3] that we will need.

Let $A$ be a unital $C^{*}$-algebra. Let $s(A)$ be the smallest positive integer $n$ for which there exists a unital *-homomorphism $A \rightarrow M_{n}$ (we set $s(A)=\infty$ if $A$ has no non-trivial finite dimensional representations). Note that if there exists a unital *-homomorphism $A \rightarrow B$ then $s(A) \leq s(B)$.

Let $A=A\left(n, d_{1}, d_{2}, \ldots, d_{N}\right)$ be a building block and let $x_{1}, x_{2}, \ldots, x_{N}$ be the exceptional points. Evaluation at $x_{i}$ gives rise to a unital *-homomorphism $\Lambda_{i}: A \rightarrow M_{d_{i}}$. This map will sometimes be denoted $\Lambda_{i}^{A}$. For every integer $k \geq 0$ we let $\Lambda_{i}^{k}$ be the direct sum of $k$ copies of the representation $\Lambda_{i}$.

Let $A=A\left(n, d_{1}, d_{2}, \ldots, d_{N}\right)$ and $B=A\left(m, e_{1}, e_{2}, \ldots, e_{M}\right)$ be building blocks. Let $\varphi: A \rightarrow B$ be a unital *-homomorphism. As in [9, Chapter 1] we define $s^{\varphi}(j, i)$ to be the multiplicity of the representation $\Lambda_{i}^{A}$ in the representation $\Lambda_{j}^{B} \circ \varphi$ for $i=1,2, \ldots, N, j=1,2, \ldots, M$.

Let us start with the $K$-theory of a building block.

Proposition 2.1. Let $A=A\left(n, d_{1}, d_{2}, \ldots, d_{N}\right)$ be a building block and let $d=\operatorname{gcd}\left(d_{1}, d_{2}, \ldots, d_{N}\right)$. We have an isomorphism of ordered groups with order units

$$
\left(K_{0}(A), K_{0}(A)^{+},[1]\right) \cong\left(\mathrm{Z}, \mathrm{Z}^{+}, d\right) .
$$

Proof. This is [3, Corollary 3.6].

Let $A=A\left(n, d_{1}, d_{2}, \ldots, d_{N}\right)$ be a building block with exceptional points $e^{2 \pi i t_{k}}, k=1,2, \ldots, N$, where $0<t_{1}<t_{2}<\ldots<t_{N}<1$. Set $t_{N+1}=t_{1}+1$ and $t_{0}=t_{N}$. Define continuous functions $\omega_{k}: \mathrm{T} \rightarrow \mathrm{T}$ for $k=1,2, \ldots, N$, by

$$
\omega_{k}\left(e^{2 \pi i t}\right)= \begin{cases}\exp \left(2 \pi i \frac{t-t_{k}}{t_{k+1}-t_{k}}\right) & t_{k} \leq t \leq t_{k+1}, \\ 1 & t_{k+1} \leq t \leq t_{k}+1 .\end{cases}
$$

Let $U_{k}^{A}$ be the unitary in $A$ defined by

$$
U_{k}^{A}(z)=\operatorname{diag}\left(\omega_{k}(z), 1,1, \ldots, 1\right), \quad z \in \mathrm{T}
$$


Set $U_{0}^{A}=U_{N}^{A}$.

Theorem 2.2. Let $A=A\left(n, d_{1}, d_{2}, \ldots, d_{N}\right)$ be a building block. Set for $k=1,2, \ldots, N-1$,

$$
s_{k}=\operatorname{lcm}\left(\frac{n}{d_{1}}, \frac{n}{d_{2}}, \ldots, \frac{n}{d_{k}}\right),
$$

and

$$
r_{k}=\operatorname{gcd}\left(s_{k}, \frac{n}{d_{k+1}}\right)=\operatorname{gcd}\left(\operatorname{lcm}\left(\frac{n}{d_{1}}, \frac{n}{d_{2}}, \ldots, \frac{n}{d_{k}}\right), \frac{n}{d_{k+1}}\right) .
$$

Choose integers $\alpha_{k}$ and $\beta_{k}$ such that

$$
r_{k}=\alpha_{k} s_{k}+\beta_{k} \frac{n}{d_{k+1}}, \quad k=1,2, \ldots, N-1 .
$$

Then

$$
K_{1}(A) \cong \mathbf{Z} \oplus \mathbf{Z}_{r_{1}} \oplus \mathbf{Z}_{r_{2}} \oplus \cdots \oplus \mathbf{Z}_{r_{N-1}} .
$$

This isomorphism can be chosen such that for $k=1,2, \ldots, N-1$, a generator of the direct summand $\mathbf{Z}_{r_{k}}$ is mapped to

$$
\left[U_{k}^{A}\right]-\frac{\beta_{k} n}{r_{k} d_{k+1}}\left[U_{k+1}^{A}\right]-\frac{\alpha_{k} s_{k}}{r_{k}}\left[U_{N}^{A}\right],
$$

and such that a generator of the direct summand $\mathrm{Z}$ is mapped to $\left[U_{N}^{A}\right]$.

Proof. See [3, Theorem 3.2].

Let $A$ and $B$ be unital $C^{*}$-algebras. A unital *-homomorphism $\varphi: A \rightarrow B$ induces morphisms $\varphi_{*}: K_{0}(A) \rightarrow K_{0}(B), \varphi_{*}: K_{1}(A) \rightarrow K_{1}(B), \varphi^{*}:$ $T(B) \rightarrow T(A), \widehat{\varphi}:$ Aff $T(A) \rightarrow$ Aff $T(B), \widetilde{\varphi}:$ Aff $T(A) / \overline{\rho_{A}\left(K_{0}(A)\right)} \rightarrow$ Aff $T(B) / \overline{\rho_{B}\left(K_{0}(B)\right)}$, and $\varphi^{\#}: U(A) / \overline{D U(A)} \rightarrow U(B) / \overline{D U(B)}$. Let $q_{A}^{\prime}$ : $U(A) \rightarrow U(A) / \overline{D U(A)}$ and $q_{A}:$ Aff $T(A) \rightarrow \operatorname{Aff} T(A) / \overline{\rho_{A}\left(K_{0}(A)\right)}$ be the canonical maps.

Proposition 2.3. Let A be a unital inductive limit of a sequence of finite direct sums of building blocks. There exists a group homomorphism

$$
\begin{aligned}
\lambda_{A}: \operatorname{Aff} T(A) / \overline{\rho_{A}\left(K_{0}(A)\right)} \rightarrow U(A) / \overline{D U(A)}, & \\
& \lambda_{A}\left(q_{A}(\widehat{a})\right)=q_{A}^{\prime}\left(e^{2 \pi i a}\right), \quad a \in A_{s a} .
\end{aligned}
$$

Let $\pi_{A}: U(A) / \overline{D U(A)} \rightarrow K_{1}(A)$ be the map induced by the canonical map $U(A) \rightarrow K_{1}(A)$. We have a short exact sequence of abelian groups

$$
0 \longrightarrow \text { Aff } T(A) / \overline{\rho_{A}\left(K_{0}(A)\right)} \stackrel{\lambda_{A}}{\longrightarrow} U(A) / \overline{D U(A)} \stackrel{\pi_{A}}{\longrightarrow} K_{1}(A) \longrightarrow 0 .
$$


This sequence is natural in A and splits unnaturally.

Proof. See [3, Proposition 5.2].

Proposition 2.4. Let $A=A\left(n, d_{1}, d_{2}, \ldots, d_{N}\right)$ be a building block. Let $u \in A$ be a unitary. Assume that

$$
\begin{aligned}
\operatorname{Det}(u(z)) & =1, \quad z \in \mathrm{T}, \\
\operatorname{Det}\left(\Lambda_{i}(u)\right) & =1, \quad i=1,2, \ldots, N .
\end{aligned}
$$

Then $u \in \overline{D U(A)}$.

Proof. See [3, Propostion 5.3].

Lemma 2.5. Let $A=A\left(n, d_{1}, d_{2}, \ldots, d_{N}\right)$ and adopt the notation of Theorem 2.2. For $k=1,2, \ldots, N-1$, there exists a unitary $v_{k}^{A} \in A$ such that $\operatorname{Det}\left(v_{k}^{A}(z)\right)=1, z \in \mathrm{T}$, and

$$
\operatorname{Det}\left(\Lambda_{j}\left(v_{k}^{A}\right)\right)= \begin{cases}\exp \left(2 \pi i \frac{\alpha_{k} s_{k}}{r_{k}} \frac{d_{j}}{n}\right) & j=1,2, \ldots, k, \\ \exp \left(-2 \pi i \frac{\beta_{k}}{r_{k}}\right) & j=k+1, \\ 1 & j=k+2, k+3, \ldots, N .\end{cases}
$$

Furthermore, $\left[v_{k}^{A}\right]$ has order $r_{k}$ in $K_{1}(A)$, and $\left[v_{1}^{A}\right],\left[v_{2}^{A}\right], \ldots,\left[v_{N-1}^{A}\right]$ generate the torsion subgroup of $K_{1}(A)$. There is a group homomorphism $\sigma_{A}$ : $\operatorname{Tor}\left(K_{1}(A)\right) \rightarrow \operatorname{Tor}(U(A) / \overline{D U(A)})$ given by $\sigma_{A}\left(\left[v_{k}^{A}\right]\right)=q_{A}^{\prime}\left(v_{k}^{A}\right), k=$ $1,2, \ldots, N-1$.

Proof. The existence of $v_{k}^{A}$ follows from [3, Lemma 5.4].

Fix $k=1,2, \ldots, N$. By [3, Lemma 5.4] there is a unitary $u \in A$ with $\operatorname{Det}(u(z))=1, z \in \mathrm{T}$, and

$$
\operatorname{Det}\left(\Lambda_{j}(u)\right)= \begin{cases}1 & j \neq k, \\ \exp \left(2 \pi i \frac{d_{k}}{n}\right) & j=k .\end{cases}
$$

Set $w=u U_{k-1}^{A} U_{k}^{A *}$. By Proposition 2.4 we have that $w$ modulo $\overline{D U(A)}$ 
equals the unitary $z \mapsto e^{2 \pi i \lambda(z)}$, where $\lambda: T \rightarrow R$ is the continuous function

$$
\lambda\left(e^{2 \pi i t}\right)= \begin{cases}\frac{1}{n} \frac{t-t_{k-1}}{t_{k}-t_{k-1}} & t_{k-1} \leq t \leq t_{k}, \\ \frac{1}{n} \frac{t_{k+1}-t}{t_{k+1}-t_{k}} & t_{k} \leq t \leq t_{k+1}, \\ 0 & \text { otherwise. }\end{cases}
$$

In particular, $w$ is trivial in $K_{1}(A)$, i.e. $[u]=\left[U_{k}^{A}\right]-\left[U_{k-1}^{A}\right]$ in $K_{1}(A)$.

As a consequence of this observation,

$$
\begin{aligned}
{\left[v_{k}^{A}\right] } & =\sum_{j=1}^{k} \frac{\alpha_{k} s_{k}}{r_{k}}\left(\left[U_{j}^{A}\right]-\left[U_{j-1}^{A}\right]\right)-\frac{\beta_{k} n}{r_{k} d_{k+1}}\left(\left[U_{k+1}^{A}\right]-\left[U_{k}^{A}\right]\right) \\
& =\left[U_{k}^{A}\right]-\frac{\beta_{k} n}{r_{k} d_{k+1}}\left[U_{k+1}^{A}\right]-\frac{\alpha_{k} s_{k}}{r_{k}}\left[U_{N}^{A}\right]
\end{aligned}
$$

in $K_{1}(A)$. Hence by Theorem 2.2 we see that $\left[v_{k}^{A}\right]$ has order $r_{k}$ in $K_{1}(A)$ and that the elements $\left[v_{1}^{A}\right],\left[v_{2}^{A}\right], \ldots,\left[v_{N-1}^{A}\right]$ generate $\operatorname{Tor}\left(K_{1}(A)\right)$. Since $r_{k} q_{A}^{\prime}\left(v_{k}^{A}\right)=$ 0 and $\pi_{A}\left(q_{A}^{\prime}\left(v_{k}^{A}\right)\right)=\left[v_{k}^{A}\right]$ it follows that $q_{A}^{\prime}\left(v_{k}^{A}\right)$ has order $r_{k}$ in $U(A) / \overline{D U(A)}$. The existence of $\sigma_{A}$ follows.

We remark that the map $\sigma_{A}$ is neither natural nor unique, and that $\pi_{A} \circ \sigma_{A}$ is the identity map on $\operatorname{Tor}\left(K_{1}(A)\right)$.

In [5] Rørdam defined the bifunctor $K L$ to be a certain quotient of $K K$. Recall from [5] that the Kasparov product yields a product $K L(B, C) \times$ $K L(A, B) \rightarrow K L(A, C)$ which we will denote by $\cdot$. Furthermore, if $K_{*}(A)$ is finitely generated then $K K(A, \cdot) \cong K L(A, \cdot)$. If $\varphi$ is a unital *-homomorphism, we let $[\varphi]$ denote the induced element in $K L(A, B)$. For unital $C^{*}-$ algebras $A$ and $B$ we let $K L(A, B)_{e}$ denote the elements of $K L(A, B)$ for which the induced map $K_{0}(A) \rightarrow K_{0}(B)$ preserves the order unit.

Let $A$ and $B$ be building blocks. $K L(A, B)$ is conveniently described in terms of the $K$-homology groups $K^{0}(A)=K K(A, \mathrm{C}) \cong K L(A, \mathrm{C})$ and $K^{0}(B)$. Recall that the Kasparov product gives rise to a group homomorphism $\kappa^{*}: K^{0}(B) \rightarrow K^{0}(A)$ for every $\kappa \in K L(A, B)$.

Theorem 2.6. Let $A=A\left(n, d_{1}, d_{2}, \ldots, d_{N}\right)$ and $B=A\left(m, e_{1}, e_{2}, \ldots, e_{M}\right)$ be building blocks such that $s(B) \geq N n$.

(i) If $v \in K L(A, B)_{e}$ then there exists a unital *-homomorphism $\varphi: A \rightarrow$ $B$ such that $[\varphi]=v$ in $K L(A, B)$. 
(ii) Let $\varphi: A \rightarrow B$ be a unital *-homomorphism and let $\kappa \in K L(A, B)_{e}$. If $\varphi^{*}=\kappa^{*}$ on $K^{0}(B)$ and $\varphi_{*}\left(\left[U_{N}^{A}\right]\right)=\kappa_{*}\left(\left[U_{N}^{A}\right]\right)$ in $K_{1}(B)$ then $[\varphi]=\kappa$ in $K L(A, B)$.

Proof. This follows from [3, Theorem 4.7].

The next result says that $K^{0}(\cdot)$ and the torsion subgroup of $U(\cdot) / \overline{D U(\cdot)}$ are related for building blocks.

Proposition 2.7. Let $A=A\left(n, d_{1}, d_{2}, \ldots, d_{N}\right)$ be a building block. There exists a finite set $F \subseteq \operatorname{Tor}(U(A) / \overline{D U(A)})$ such that if $B$ is a building block and $\varphi, \psi: A \rightarrow B$ are unital $*$-homomorphisms with $\varphi^{\#}(x)=\varphi^{\#}(x), x \in F$, then $\varphi^{*}=\psi^{*}$ on $K^{0}(B)$.

Proof. This is part of [3, Theorem 5.5].

We also need a description of the structure of the group $K^{0}(\cdot)$ for a building block.

Proposition 2.8. Let $A=A\left(n, d_{1}, d_{2}, \ldots, d_{N}\right)$ be a building block. Then $K^{0}(A)$ is generated by $\left[\Lambda_{1}\right],\left[\Lambda_{2}\right], \ldots,\left[\Lambda_{N}\right]$. Furthermore, for $a_{1}, a_{2}$, $\ldots, a_{N} \in \mathrm{Z}$ we have that

$$
a_{1}\left[\Lambda_{1}\right]+a_{2}\left[\Lambda_{2}\right]+\cdots+a_{N}\left[\Lambda_{N}\right]=0
$$

if and only if there exist $b_{1}, b_{2}, \ldots, b_{N} \in \mathbf{Z}$ such that $\sum_{i=1}^{N} b_{i}=0$ and

$$
a_{i}=b_{i} \frac{n}{d_{i}}, \quad i=1,2, \ldots, N .
$$

Proof. This is [3, Proposition 4.2].

We conclude with a technical proposition which is needed in the next section.

Proposition 2.9. Let $A=A\left(n, d_{1}, d_{2}, \ldots, d_{N}\right)$ and $B=A\left(m, e_{1}, e_{2}\right.$, $\left.\ldots, e_{M}\right)$ be building blocks with $s(B) \geq N n$. Let $\chi \in K_{1}(B)$ and let $h$ : $K^{0}(B) \rightarrow K^{0}(A)$ be a homomorphism of the form

$$
\left(\begin{array}{c}
h\left(\left[\Lambda_{1}^{B}\right]\right) \\
h\left(\left[\Lambda_{2}^{B}\right]\right) \\
\vdots \\
h\left(\left[\Lambda_{M}^{B}\right]\right)
\end{array}\right)=\left(\begin{array}{cccc}
h_{11} & h_{12} & \ldots & h_{1 N} \\
h_{21} & h_{22} & \ldots & h_{2 N} \\
\vdots & \vdots & & \vdots \\
h_{M 1} & h_{M 2} & \ldots & h_{M N}
\end{array}\right)\left(\begin{array}{c}
{\left[\Lambda_{1}^{A}\right]} \\
{\left[\Lambda_{2}^{A}\right]} \\
\vdots \\
{\left[\Lambda_{N}^{A}\right]}
\end{array}\right)
$$

with $\sum_{i=1}^{N} h_{j i} d_{i}=e_{j}$ for $j=1,2, \ldots, M$. There exists a unital *-homomorphism $\varphi: A \rightarrow B$ such that $\varphi^{*}=h$ on $K^{0}(B)$ and $\varphi_{*}\left(\left[U_{N}^{A}\right]\right)=\chi$ in $K_{1}(B)$. 
Proof. By Proposition 2.8 we have that $\frac{n}{d_{i}}\left[\Lambda_{i}^{A}\right]=\frac{n}{d_{N}}\left[\Lambda_{N}^{A}\right]$ in $K^{0}(A)$. Hence we may assume that $0 \leq h_{j i}<\frac{n}{d_{i}}$ for $i \neq N$ and still have that $\sum_{i=1}^{N} h_{j i} d_{i}=e_{j}$ for $j=1,2, \ldots, M$. Note that for $j=1,2, \ldots, M$,

$$
N n \leq \sum_{i=1}^{N} h_{j i} d_{i}<(N-1) n+h_{j N} d_{N}
$$

and hence $h_{j N}>\frac{n}{d_{N}}$. The conclusion follows from [3, Proposition 4.4].

\section{3. $K L$ and other invariants}

Let $A$ and $B$ be unital $C^{*}$-algebras and let $\varphi_{0}: K_{0}(A) \rightarrow K_{0}(B)$ be an order unit preserving group homomorphism. Assume that $K_{0}(A) \cong Z$. Then $\rho_{A}\left(K_{0}(A)\right)$ is closed in Aff $T(A)$, and we have a well-defined map

$$
\widetilde{\varphi_{0}}: \operatorname{Tor}\left(\operatorname{Aff} T(A) / \overline{\rho_{A}\left(K_{0}(A)\right)}\right) \rightarrow \operatorname{Tor}\left(\operatorname{Aff} T(B) / \overline{\rho_{B}\left(K_{0}(B)\right)}\right)
$$

given by $\widetilde{\varphi}_{0}\left(q_{A}\left(\frac{1}{k} \rho_{A}(x)\right)\right)=q_{B}\left(\frac{1}{k} \rho_{B}\left(\varphi_{0}(x)\right)\right)$ for $x \in K_{0}(A)$ and every positive integer $k$.

Theorem 3.1. Let $A=A\left(n, d_{1}, d_{2}, \ldots, d_{N}\right)$ and $B=A\left(m, e_{1}, e_{2}, \ldots, e_{M}\right)$ be building blocks with $s(B) \geq N n$. If $\varphi_{0}: K_{0}(A) \rightarrow K_{0}(B)$ is an order unit preserving group homomorphism, if $\Phi: \operatorname{Tor}(U(A) / \overline{D U(A)}) \rightarrow$ $\operatorname{Tor}(U(B) / \overline{D U(B)})$ is a group homomorphism such that the diagram

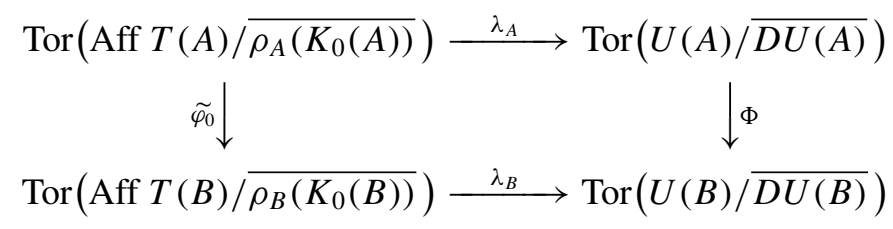

commutes, and if $\chi \in K_{1}(B)$, then there is a unital $*$-homomorphism $\psi: A \rightarrow$ $B$ such that $\psi^{\#}=\Phi$ on $\operatorname{Tor}(U(A) / \overline{D U(A)})$ and $\psi_{*}\left[U_{N}^{A}\right]=\chi$ in $K_{1}(B)$.

Proof. We adopt the notation of Theorem 2.2. Set $\alpha_{0}=1$. If $i$ and $k$ are integers, $1 \leq i \leq k \leq N$, we define an integer $c_{i}^{k}$ by

$$
c_{i}^{k}=\alpha_{i-1} \beta_{i} \beta_{i+1} \ldots \beta_{k-1} .
$$

We claim that

$$
\frac{1}{s_{k}}=\sum_{i=1}^{k} c_{i}^{k} \frac{d_{i}}{n}, \quad k=1,2, \ldots, N
$$


As $c_{1}^{1}=1$, this is clear for $k=1$. Assume it is correct for $k, 1 \leq k \leq N-1$. Then

$$
\begin{aligned}
\sum_{i=1}^{k+1} c_{i}^{k+1} \frac{d_{i}}{n} & =c_{k+1}^{k+1} \frac{d_{k+1}}{n}+\sum_{i=1}^{k} c_{i}^{k+1} \frac{d_{i}}{n}=c_{k+1}^{k+1} \frac{d_{k+1}}{n}+\beta_{k} \sum_{i=1}^{k} c_{i}^{k} \frac{d_{i}}{n} \\
& =\alpha_{k} \frac{d_{k+1}}{n}+\beta_{k} \frac{1}{s_{k}}=\frac{d_{k+1}}{n} \frac{1}{s_{k}}\left(\alpha_{k} s_{k}+\beta_{k} \frac{n}{d_{k+1}}\right) \\
& =\frac{r_{k}}{s_{k} \frac{n}{d_{k+1}}}=\frac{1}{s_{k+1}},
\end{aligned}
$$

proving (1).

Choose a unitary $u_{k} \in B$ such that $\Phi\left(q_{A}^{\prime}\left(v_{k}^{A}\right)\right)=q_{B}^{\prime}\left(u_{k}\right), k=1,2, \ldots, N-$ 1. Let $q_{k}^{j} \in \mathrm{R}$ be numbers such that

$$
\operatorname{Det}\left(\Lambda_{j}\left(u_{k}\right)\right)=e^{2 \pi i q_{k}^{j}}, \quad k=1,2, \ldots, N-1, j=1,2, \ldots, M .
$$

Set $q_{0}^{j}=0$ and set $d=\operatorname{gcd}\left(d_{1}, d_{2}, \ldots, d_{N}\right)$. By Proposition $2.1 d$ divides $e_{j}$, $j=1,2, \ldots, M$. Define for $i=1,2, \ldots, N, j=1,2, \ldots, M$,

$$
h_{j i}=c_{i}^{N} \frac{e_{j}}{d}-\frac{n}{d_{i}} q_{i-1}^{j}+\sum_{l=1}^{N-i} c_{i}^{N-l} s_{N-l} q_{N-l}^{j} .
$$

Since $r_{k} q_{A}^{\prime}\left(v_{k}^{A}\right)=0$ in $U(A) / \overline{D U(A)}$ by Lemma 2.5 , we see that $u_{k}^{r_{k}} \in$ $\overline{D U(B)}$ and hence $r_{k} q_{k}^{j} \in \mathrm{Z}, k=1,2, \ldots, N-1$. It follows that $h_{j i} \in \mathbf{Z}$ for every $i$ and $j$. Since $q_{B}^{\prime}\left(u_{k}\right)$ has finite order, $\operatorname{Det}\left(u_{k}(\cdot)\right)$ is constantly equal to $e^{2 \pi i a_{k}}$ for some $a_{k} \in \mathrm{R}$. Note that

$$
e^{2 \pi i a_{k}}=e^{2 \pi i q_{k}^{j} \frac{m}{e_{j}}}, \quad j=1,2, \ldots, M, k=1,2, \ldots, N-1 .
$$

Thus if we set $a_{0}=0$ we find that

$$
\begin{aligned}
\frac{m}{e_{j}} h_{j i} & =c_{i}^{N} \frac{m}{d}-\frac{n}{d_{i}} q_{i-1}^{j} \frac{m}{e_{j}}+\sum_{l=1}^{N-i} c_{i}^{N-l} s_{N-l} q_{N-l}^{j} \frac{m}{e_{j}} \\
& \equiv c_{i}^{N} \frac{m}{d}-\frac{n}{d_{i}} a_{i-1}+\sum_{l=1}^{N-i} c_{i}^{N-l} s_{N-l} a_{N-l} \quad \bmod \frac{n}{d_{i}}
\end{aligned}
$$

for $i=1,2, \ldots, N, j=1,2, \ldots, M$. Hence

$$
\frac{m}{e_{j}} h_{j i} \equiv \frac{m}{e_{M}} h_{M i} \quad \bmod \frac{n}{d_{i}} .
$$


For $k=1,2, \ldots, N$,

$$
\begin{aligned}
\sum_{i=1}^{k} \sum_{l=1}^{N-i} c_{i}^{N-l} s_{N-l} q_{N-l}^{j} \frac{d_{i}}{n} \\
\quad=\sum_{l=1}^{N-1} \sum_{i=1}^{\min (k, N-l)} c_{i}^{N-l} s_{N-l} q_{N-l}^{j} \frac{d_{i}}{n} \\
=\sum_{l=1}^{N-k} \sum_{i=1}^{k} c_{i}^{N-l} s_{N-l} q_{N-l}^{j} \frac{d_{i}}{n}+\sum_{l=N-k+1}^{N-1} \sum_{i=1}^{N-l} c_{i}^{N-l} s_{N-l} q_{N-l}^{j} \frac{d_{i}}{n} \\
=\sum_{l=1}^{N-k} \beta_{k} \beta_{k+1} \ldots \beta_{N-l-1} \sum_{i=1}^{k} c_{i}^{k} \frac{d_{i}}{n} s_{N-l} q_{N-l}^{j}+\sum_{l=N-k+1}^{N-1} q_{N-l}^{j} \\
=\sum_{l=1}^{N-k} \beta_{k} \beta_{k+1} \ldots \beta_{N-l-1} \frac{1}{s_{k}} s_{N-l} q_{N-l}^{j}+\sum_{l=1}^{k-1} q_{l}^{j} .
\end{aligned}
$$

Hence

$$
\begin{aligned}
\sum_{i=1}^{k} h_{j i} \frac{d_{i}}{n} & =\sum_{i=1}^{k} c_{i}^{N} \frac{e_{j}}{d} \frac{d_{i}}{n}-\sum_{i=1}^{k} q_{i-1}^{j}+\sum_{i=1}^{k} \sum_{l=1}^{N-i} c_{i}^{N-l} s_{N-l} q_{N-l}^{j} \frac{d_{i}}{n} \\
& =\frac{e_{j}}{d} \beta_{k} \beta_{k+1} \ldots \beta_{N-1} \sum_{i=1}^{k} c_{i}^{k} \frac{d_{i}}{n}+\sum_{l=1}^{N-k} \beta_{k} \beta_{k+1} \ldots \beta_{N-l-1} \frac{1}{s_{k}} s_{N-l} q_{N-l}^{j} \\
& =\frac{e_{j}}{d} \beta_{k} \beta_{k+1} \ldots \beta_{N-1} \frac{1}{s_{k}}+\sum_{l=1}^{N-k} \beta_{k} \beta_{k+1} \ldots \beta_{N-l-1} \frac{1}{s_{k}} s_{N-l} q_{N-l}^{j} .
\end{aligned}
$$

By setting $k=N$ we see that

$$
\sum_{i=1}^{N} h_{j i} d_{i}=e_{j} \frac{n}{d} \frac{1}{s_{N}}=e_{j} .
$$

Combining this equation with (2) and Proposition 2.8 it is an elementary exercise to prove that we can define a homomorphism $h: K^{0}(B) \rightarrow K^{0}(A)$ by

$$
\left(\begin{array}{c}
h\left(\left[\Lambda_{1}^{B}\right]\right) \\
h\left(\left[\Lambda_{2}^{B}\right]\right) \\
\vdots \\
h\left(\left[\Lambda_{M}^{B}\right]\right)
\end{array}\right)=\left(\begin{array}{cccc}
h_{11} & h_{12} & \ldots & h_{1 N} \\
h_{21} & h_{22} & \ldots & h_{2 N} \\
\vdots & \vdots & & \vdots \\
h_{M 1} & h_{M 2} & \ldots & h_{M N}
\end{array}\right)\left(\begin{array}{c}
{\left[\Lambda_{1}^{A}\right]} \\
{\left[\Lambda_{2}^{A}\right]} \\
\vdots \\
{\left[\Lambda_{N}^{A}\right]}
\end{array}\right) .
$$


By Proposition 2.9 there exists a unital *-homomorphism $\psi: A \rightarrow B$ such that $\psi^{*}=h$ on $K^{0}(B)$ and $\psi_{*}\left(\left[U_{N}^{A}\right]\right)=\chi$ on $K_{1}(B)$. Fix $j=1,2, \ldots, M$. Let $t_{i}=s^{\psi}(j, i)$. By [3, Lemma 2.1] there exist a unitary $w \in M_{e_{j}}$ and $z_{1}, z_{2}, \ldots, z_{L} \in \mathrm{T}$ such that

$\Lambda_{j}^{B} \circ \psi(f)$

$$
=w \operatorname{diag}\left(\Lambda_{1}^{t_{1}}(f), \Lambda_{2}^{t_{2}}(f), \ldots, \Lambda_{N}^{t_{N}}(f), f\left(z_{1}\right), f\left(z_{2}\right), \ldots, f\left(z_{L}\right)\right) w^{*}
$$

for $f \in A$. Since point-evaluations are homotopic*-homomorphisms $A \rightarrow$ $M_{n}$, we see that

$$
\psi^{*}\left[\Lambda_{j}^{B}\right]=\left[\Lambda_{j}^{B} \circ \psi\right]=\sum_{i=1}^{N} t_{i}\left[\Lambda_{i}^{A}\right]+L \frac{n}{d_{N}}\left[\Lambda_{N}^{A}\right] .
$$

in $K^{0}(A)$. On the other hand, $\psi^{*}\left[\Lambda_{j}^{B}\right]=\sum_{i=1}^{N} h_{j i}\left[\Lambda_{i}^{A}\right]$. It follows from Proposition 2.8 that

$$
s^{\psi}(j, i) \equiv h_{j i} \quad \bmod \frac{n}{d_{i}}, \quad i=1,2, \ldots, N, \quad j=1,2, \ldots, M .
$$

Note that for $k=1,2, \ldots, N-1, j=1,2, \ldots, M$,

$$
\begin{aligned}
\frac{\alpha_{k} s_{k}}{r_{k}} \sum_{i=1}^{k} h_{j i} \frac{d_{i}}{n} & =\frac{e_{j}}{d} \frac{\alpha_{k}}{r_{k}} \beta_{k} \beta_{k+1} \ldots \beta_{N-1}+\sum_{l=1}^{N-k} \frac{\alpha_{k}}{r_{k}} \beta_{k} \beta_{k+1} \ldots \beta_{N-l-1} s_{N-l} q_{N-l}^{j} \\
& =\frac{e_{j}}{d} \frac{\beta_{k}}{r_{k}} c_{k+1}^{N}+\sum_{l=1}^{N-k-1} \frac{\beta_{k}}{r_{k}} c_{k+1}^{N-l} s_{N-l} q_{N-l}^{j}+\frac{\alpha_{k}}{r_{k}} s_{k} q_{k}^{j} \\
& =\frac{\beta_{k}}{r_{k}}\left(h_{j(k+1)}+\frac{n}{d_{k+1}} q_{k}^{j}\right)+\frac{\alpha_{k}}{r_{k}} s_{k} q_{k}^{j} \\
& =\frac{\beta_{k}}{r_{k}} h_{j(k+1)}+q_{k}^{j} .
\end{aligned}
$$

Since $\operatorname{Det}\left(v_{k}^{A}(z)\right)=1, z \in \mathrm{T}$, we see that

$$
\begin{aligned}
\operatorname{Det}\left(\Lambda_{j}\left(\psi\left(v_{k}^{A}\right)\right)\right) & =\prod_{i=1}^{N} \operatorname{Det}\left(\Lambda_{i}\left(v_{k}^{A}\right)\right)^{s^{\psi}(j, i)}=\prod_{i=1}^{N} \operatorname{Det}\left(\Lambda_{i}\left(v_{k}^{A}\right)\right)^{h(j, i)} \\
& =\exp \left(2 \pi i\left(\sum_{i=1}^{k} \frac{\alpha_{k} s_{k}}{r_{k}} h_{j i} \frac{d_{i}}{n}-\frac{\beta_{k}}{r_{k}} h_{j(k+1)}\right)\right) \\
& =\exp \left(2 \pi i q_{k}^{j}\right)=\operatorname{Det}\left(\Lambda_{j}\left(u_{k}\right)\right) .
\end{aligned}
$$


Thus $\operatorname{Det}\left(\psi\left(v_{k}^{A}\right)(\cdot)\right)$ and $\operatorname{Det}\left(u_{k}(\cdot)\right)$ agree at the exceptional points of $B$, and hence they agree everywhere. It follows from Proposition 2.4 that

$$
q_{B}^{\prime}\left(\psi\left(v_{k}^{A}\right)\right)=q_{B}^{\prime}\left(u_{k}\right)=\Phi\left(q_{A}^{\prime}\left(v_{k}^{A}\right)\right), \quad k=1,2, \ldots, N-1 .
$$

As $\tilde{\psi}=\tilde{\varphi}_{0}$ on $\operatorname{Tor}\left(\operatorname{Aff} T(A) / \overline{\rho_{A}\left(K_{0}(A)\right)}\right)$, we conclude from Lemma 2.5 and Proposition 2.3 that $\psi^{\#}$ and $\Phi$ agree on all of $\operatorname{Tor}(U(A) / \overline{D U(A)})$.

Our next result says that the information contained in $K L(A, B)$ can be detected by other invariants when $A$ and $B$ are building blocks.

Proposition 3.2. Let $A=A\left(n, d_{1}, d_{2}, \ldots, d_{N}\right)$ and $B$ be building blocks with $s(B) \geq N n$. Let $\varphi_{0}: K_{0}(A) \rightarrow K_{0}(B)$ be an order unit preserving group homomorphism, and let $\Phi: \operatorname{Tor}(U(A) / \overline{D U(A)}) \rightarrow \operatorname{Tor}(U(B) / \overline{D U(B)})$ and $\varphi_{1}: K_{1}(A) \rightarrow K_{1}(B)$ be group homomorphisms such that the diagram $\operatorname{Tor}\left(\right.$ Aff $\left.T(A) / \overline{\rho_{A}\left(K_{0}(A)\right)}\right) \stackrel{\lambda_{A}}{\longrightarrow} \operatorname{Tor}(U(A) / \overline{D U(A)}) \stackrel{\pi_{A}}{\longrightarrow} \operatorname{Tor}\left(K_{1}(A)\right)$

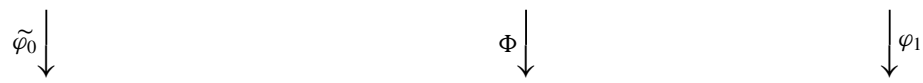

$\operatorname{Tor}\left(\operatorname{Aff} T(B) / \overline{\rho_{B}\left(K_{0}(B)\right)}\right) \underset{\lambda_{B}}{\longrightarrow} \operatorname{Tor}(U(B) / \overline{D U(B)}) \underset{\pi_{B}}{\longrightarrow} \operatorname{Tor}\left(K_{1}(B)\right)$ commutes.

(i) There exists a unital $*$-homomorphism $\varphi: A \rightarrow B$ such that $\varphi_{*}=\varphi_{0}$ on $K_{0}(A), \varphi_{*}=\varphi_{1}$ on $K_{1}(A)$ and $\varphi^{\#}=\Phi$ on $\operatorname{Tor}(U(A) / \overline{D U(A)})$.

(ii) If $\psi: A \rightarrow B$ is another unital $*_{-}$homomorphism such that $\psi_{*}=\varphi_{0}$ on $K_{0}(A), \psi_{*}=\varphi_{1}$ on $K_{1}(A)$ and $\psi^{\#}=\Phi$ on $\operatorname{Tor}(U(A) / \overline{D U(A)})$, then $[\varphi]=[\psi]$ in $K L(A, B)$.

Proof. Choose by Theorem 3.1 a unital ${ }^{*}$-homomorphism $\varphi: A \rightarrow B$ such that $\varphi^{\#}=\Phi$ on $\operatorname{Tor}(U(A) / \overline{D U(A)})$ and $\varphi_{*}\left[U_{N}^{A}\right]=\varphi_{1}\left[U_{N}^{A}\right]$ in $K_{1}(B)$. Then $\varphi_{*}=\varphi_{1}$ on $\operatorname{Tor}\left(K_{1}(A)\right)$, and thus $\varphi_{*}=\varphi_{1}$ on all of $K_{1}(A)$ by Theorem 2.2. Obviously $\varphi_{*}=\varphi_{0}$ since $\varphi$ is unital. This proves (i).

To prove (ii), note that since $\varphi^{\#}=\psi^{\#}$ on $\operatorname{Tor}(U(A) / \overline{D U(A)})$ we have that $\varphi^{*}=\psi^{*}$ on $K^{0}(B)$ by Proposition 2.7. Hence $[\varphi]=[\psi]$ by Theorem 2.6.

Let $A$ and $B$ be simple unital infinite dimensional inductive limits of sequences of finite direct sums of building blocks. In [3, Chapter 10] a group homomorphism

$$
s_{\kappa}: \operatorname{Tor}(U(A) / \overline{D U(A)}) \rightarrow \operatorname{Tor}(U(B) / \overline{D U(B)}),
$$

was constructed for every $\kappa \in K L(A, B)_{T}$ (the map was constructed for slightly different $B$ but can be applied in our case by [3, Lemma 10.3], [3, Lemma 9.6] and [3, Theorem 9.9]). Recall from [3] that $K L(A, B)_{T}$ is the 
set of elements $\kappa \in K L(A, B)_{e}$ for which there exists an affine continuous map $\varphi_{T}: T(B) \rightarrow T(A)$ such that $r_{B}(\omega)\left(\kappa_{*}(x)\right)=r_{A}\left(\varphi_{T}(\omega)\right)(x)$ for $x \in K_{0}(A), \omega \in T(B)$. Note that if $K_{0}(A) \cong \mathrm{Z}$ and $K_{0}(B) \cong \mathrm{Z}$ then $K L(A, B)_{T}=K L(A, B)_{e}$.

Recall furthermore from [3, Chapter 10] that $s_{[\mu]}=\mu^{\#}$ on $\operatorname{Tor}(U(A) /$ $\overline{D U(A)})$ for every unital *-homomorphism $\mu: A \rightarrow B$, and that if $C$ is a finite direct sum of building blocks, and $\varphi: C \rightarrow A, \psi: C \rightarrow B$ are unital *-homomorphisms such that $[\psi]=\kappa \cdot[\varphi]$ in $K L(C, B)$, then $\psi^{\#}=s_{\kappa} \circ \varphi^{\#}$ on $\operatorname{Tor}(U(C) / \overline{D U(C)})$.

We can now generalize Theorem 3.2 to simple inductive limits for which $K_{0}(A)$ and $K_{0}(B)$ are cyclic:

THEOREM 3.3. Let $A$ and $B$ be unital simple infinite dimensional inductive limits of sequences of finite direct sums of building blocks. Assume that $K_{0}(A)$ and $K_{0}(B)$ are cyclic groups. Let $\varphi_{0}: K_{0}(A) \rightarrow K_{0}(B)$ be an order unit preserving group homomorphism, and let $\varphi_{1}: K_{1}(A) \rightarrow K_{1}(B)$ and $\Phi:$ $U(A) / \overline{D U(A)} \rightarrow U(B) / \overline{D U(B)}$ be group homomorphisms such that the diagram

$\operatorname{Tor}\left(\operatorname{Aff} T(A) / \overline{\rho_{A}\left(K_{0}(A)\right)}\right) \stackrel{\lambda_{A}}{\longrightarrow} \operatorname{Tor}(U(A) / \overline{D U(A)}) \stackrel{\pi_{A}}{\longrightarrow} \operatorname{Tor}\left(K_{1}(A)\right)$

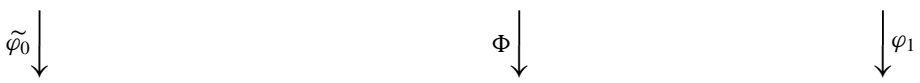

$\operatorname{Tor}\left(\operatorname{Aff} T(B) / \overline{\rho_{B}\left(K_{0}(B)\right)}\right) \underset{\lambda_{B}}{\longrightarrow} \operatorname{Tor}(U(B) / \overline{D U(B)}) \underset{\pi_{B}}{\longrightarrow} \operatorname{Tor}\left(K_{1}(B)\right)$

commutes. There exists a unique element $\kappa \in K L(A, B)$ such that $\kappa_{*}=\varphi_{0}$ on $K_{0}(A), \kappa_{*}=\varphi_{1}$ on $K_{1}(A)$ and $s_{\kappa}=\Phi$ on $\operatorname{Tor}(U(A) / \overline{D U(A)})$.

Proof. We may by [3, Theorem 9.9] assume that $A$ is the inductive limit of a sequence

$$
A_{1} \stackrel{\alpha_{1}}{\longrightarrow} A_{2} \stackrel{\alpha_{2}}{\longrightarrow} A_{3} \stackrel{\alpha_{3}}{\longrightarrow} \ldots
$$

of finite direct sums of building blocks with unital and injective connecting maps. Similarly we may assume that $B$ is the inductive limit of a sequence

$$
B_{1} \stackrel{\beta_{1}}{\longrightarrow} B_{2} \stackrel{\beta_{2}}{\longrightarrow} B_{3} \stackrel{\beta_{3}}{\longrightarrow} \ldots
$$

of finite direct sums of building blocks with unital and injective connecting maps. Since $K_{0}(A) \cong \mathrm{Z}$ it is easy to see that we may furthermore assume that each $A_{k}$ is a building block, rather than a finite direct sum of building blocks. Similarly we may assume that each $B_{k}$ is a building block. Let $\alpha_{k, \infty}: A_{k} \rightarrow A$ and $\beta_{k, \infty}: B_{k} \rightarrow B$ denote the canonical *-homomorphisms.

By passing to subsequences we may assume that for every positive integer $k$ there exist an order unit preserving group homomorphism $\mu_{k}: K_{0}\left(A_{k}\right) \rightarrow$ 
$K_{0}\left(B_{k}\right)$ and a group homomorphism $\eta_{k}: K_{1}\left(A_{k}\right) \rightarrow K_{1}\left(B_{k}\right)$ such that

$$
\begin{array}{lll}
\beta_{k, \infty_{*}} \circ \mu_{k}=\varphi_{0} \circ \alpha_{k, \infty_{*}} & \text { on } & K_{0}\left(A_{k}\right), \\
\beta_{k, \infty_{*}} \circ \eta_{k}=\varphi_{1} \circ \alpha_{k, \infty_{*}} & \text { on } & K_{1}\left(A_{k}\right) .
\end{array}
$$

By passing to a subsequence again, we may assume that

$$
\begin{aligned}
& \mu_{k+1} \circ \alpha_{k *}=\beta_{k_{*}} \circ \mu_{k} \quad \text { on } \quad K_{0}\left(A_{k}\right) \text {, } \\
& \eta_{k+1} \circ \alpha_{k *}=\beta_{k *} \circ \eta_{k} \quad \text { on } \quad K_{1}\left(A_{k}\right) \text {. }
\end{aligned}
$$

Let $A_{k}=A\left(n_{k}, d_{1}^{k}, d_{2}^{k}, \ldots, d_{N_{k}}^{k}\right)$. By Proposition 2.3, Lemma 2.5, and [3, Lemma 10.8], we may also assume that for every positive integer $k$, there exists a group homomorphism $\Phi_{k}: \operatorname{Tor}\left(U\left(A_{k}\right) / \overline{D U\left(A_{k}\right)}\right) \rightarrow \operatorname{Tor}\left(U\left(B_{k}\right) / \overline{D U\left(B_{k}\right)}\right)$ such that

$$
\lambda_{B_{k}} \circ \widetilde{\mu_{k}}=\Phi_{k} \circ \lambda_{A_{k}}
$$

on $\operatorname{Tor}\left(\operatorname{Aff} T\left(A_{k}\right) / \overline{\rho_{A_{k}}\left(K_{0}\left(A_{k}\right)\right)}\right)$ and

$$
\beta_{k, \infty}^{\#} \circ \Phi_{k}\left(q_{A_{k}}^{\prime}\left(v_{j}^{A_{k}}\right)\right)=\Phi \circ \alpha_{k, \infty}^{\#}\left(q_{A_{k}}^{\prime}\left(v_{j}^{A_{k}}\right)\right)
$$

for $j=1,2, \ldots, N_{k}-1$. Since for every positive integer $k$,

$$
\begin{aligned}
\beta_{k, \infty}^{\#} \circ \Phi_{k} \circ \lambda_{A_{k}} & =\beta_{k, \infty}^{\#} \circ \lambda_{B_{k}} \circ \widetilde{\mu_{k}}=\lambda_{B} \circ \widetilde{\beta_{k, \infty}} \circ \widetilde{\mu_{k}} \\
& =\lambda_{B} \circ \widetilde{\varphi_{0}} \circ \widetilde{\alpha_{k, \infty}}=\Phi \circ \lambda_{A} \circ \widetilde{\alpha_{k, \infty}}=\Phi \circ \alpha_{k, \infty}^{\#} \circ \lambda_{A_{k}}
\end{aligned}
$$

on Tor(Aff $T\left(A_{k}\right) / \overline{\rho_{A_{k}}\left(K_{0}\left(A_{k}\right)\right)}$ ), we conclude from Proposition 2.3 and Lemma 2.5 that

$$
\beta_{k, \infty}^{\#} \circ \Phi_{k}=\Phi \circ \alpha_{k, \infty}^{\#}
$$

on $\operatorname{Tor}\left(U\left(A_{k}\right) / \overline{D U\left(A_{k}\right)}\right)$.

It follows from the above equation and [3, Lemma 10.4] that by passing to subsequences we may assume that for every positive integer $k$,

$$
\beta_{k}^{\#} \circ \Phi_{k}\left(q_{A_{k}}^{\prime}\left(v_{j}^{A_{k}}\right)\right)=\Phi_{k+1} \circ \alpha_{k}^{\#}\left(q_{A_{k}}^{\prime}\left(v_{j}^{A_{k}}\right)\right)
$$

for $j=1,2, \ldots, N_{k}-1$. Since for every positive integer $k$,

$$
\begin{aligned}
\beta_{k}^{\#} \circ \Phi_{k} \circ \lambda_{A_{k}} & =\beta_{k}^{\#} \circ \lambda_{B_{k}} \circ \widetilde{\mu_{k}}=\lambda_{B_{k+1}} \circ \widetilde{\beta_{k}} \circ \widetilde{\mu_{k}} \\
& =\lambda_{B_{k+1}} \circ \widetilde{\mu_{k+1}} \circ \widetilde{\alpha_{k}}=\Phi_{k+1} \circ \lambda_{A_{k+1}} \circ \widetilde{\alpha_{k}}=\Phi_{k+1} \circ \alpha_{k}^{\#} \circ \lambda_{A_{k}}
\end{aligned}
$$

on Tor(Aff $T\left(A_{k}\right) / \overline{\rho_{A_{k}}\left(K_{0}\left(A_{k}\right)\right)}$ ), we see that

$$
\beta_{k}^{\#} \circ \Phi_{k}=\Phi_{k+1} \circ \alpha_{k}^{\#}
$$


on $\operatorname{Tor}\left(U\left(A_{k}\right) / \overline{D U\left(A_{k}\right)}\right)$.

Note that for every positive integer $k$,

$$
\begin{aligned}
\beta_{k, \infty_{*}} \circ \eta_{k} \circ \pi_{A_{k}} & =\varphi_{1} \circ \alpha_{k, \infty_{*}} \circ \pi_{A_{k}}=\varphi_{1} \circ \pi_{A} \circ \alpha_{k, \infty}^{\#} \\
& =\pi_{B} \circ \Phi \circ \alpha_{k, \infty}^{\#}=\pi_{B} \circ \beta_{k, \infty}^{\#} \circ \Phi_{k}=\beta_{k, \infty_{*}} \circ \pi_{B_{k}} \circ \Phi_{k}
\end{aligned}
$$

on $\operatorname{Tor}\left(U\left(A_{k}\right) / \overline{D U\left(A_{k}\right)}\right)$. By passing to subsequences again we may assume that

$$
\eta_{k} \circ \pi_{A_{k}}\left(q_{A_{k}}^{\prime}\left(v_{j}^{A_{k}}\right)\right)=\pi_{B_{k}} \circ \Phi_{k}\left(q_{A_{k}}^{\prime}\left(v_{j}^{A_{k}}\right)\right)
$$

in $K_{1}(B)$ for $j=1,2, \ldots, N_{k}-1$. Since $\pi_{B_{k}} \circ \Phi_{k} \circ \lambda_{A_{k}}=0$ on the torsion subgroup of Aff $T\left(A_{k}\right) / \overline{\rho_{A_{k}}\left(K_{0}\left(A_{k}\right)\right)}$, it follows that $\eta_{k} \circ \pi_{A_{k}}=\pi_{B_{k}} \circ \Phi_{k}$ on $\operatorname{Tor}\left(U\left(A_{k}\right) / \overline{D U\left(A_{k}\right)}\right)$. Thus the diagram

$\operatorname{Tor}\left(\operatorname{Aff} T\left(A_{k}\right) / \overline{\rho_{A_{k}}\left(K_{0}\left(A_{k}\right)\right)}\right) \stackrel{\lambda_{A_{k}}}{\longrightarrow} \operatorname{Tor}\left(U\left(A_{k}\right) / \overline{D U\left(A_{k}\right)}\right) \stackrel{\pi_{A_{k}}}{\longrightarrow} \operatorname{Tor}\left(K_{1}\left(A_{k}\right)\right)$

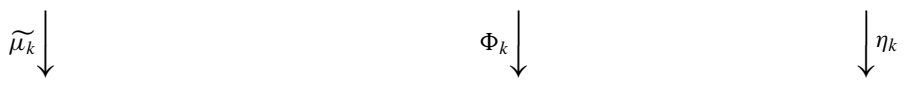

$\operatorname{Tor}\left(\operatorname{Aff} T\left(B_{k}\right) / \overline{\rho_{B_{k}}\left(K_{0}\left(B_{k}\right)\right)}\right) \underset{\lambda_{B_{k}}}{\longrightarrow} \operatorname{Tor}\left(U\left(B_{k}\right) / \overline{D U\left(B_{k}\right)}\right) \underset{\pi_{B_{k}}}{\longrightarrow} \operatorname{Tor}\left(K_{1}\left(B_{k}\right)\right)$

commutes. Finally we may by [3, Lemma 9.6] assume that $s\left(B_{k}\right) \geq N_{k} n_{k}$.

It follows from Proposition 3.2 that for every positive integer $k$, there exists a unital ${ }^{*}$-homomorphism $\psi_{k}: A_{k} \rightarrow B_{k}$ such that $\psi_{k_{*}}=\mu_{k}$ on $K_{0}\left(A_{k}\right), \psi_{k *}=$ $\eta_{k}$ on $K_{1}\left(A_{k}\right)$, and $\psi_{k}^{\#}=\Phi_{k}$ on $\operatorname{Tor}\left(U\left(A_{k}\right) / \overline{D U\left(A_{k}\right)}\right)$. By the uniqueness part of the same proposition, $\left[\beta_{k}\right] \cdot\left[\psi_{k}\right]=\left[\psi_{k+1}\right] \cdot\left[\alpha_{k}\right]$ in $K L\left(A_{k}, B_{k+1}\right)$. By [6, Theorem 1.12] and [7, Theorem 7.1] there exists an element $\kappa \in K L(A, B)$ such that $\kappa \cdot\left[\alpha_{k, \infty}\right]=\left[\beta_{k, \infty}\right] \cdot\left[\psi_{k}\right]$ in $K L\left(A_{k}, B\right)$ for every positive integer $k$. Then $\kappa_{*}=\varphi_{0}$ on $K_{0}(A), \kappa_{*}=\varphi_{1}$ on $K_{1}(A)$, and

$s_{\kappa} \circ \alpha_{k, \infty}^{\#}=\left(\beta_{k, \infty} \circ \psi_{k}\right)^{\#}=\beta_{k, \infty}^{\#} \circ \Phi_{k}=\Phi \circ \alpha_{k, \infty}^{\#} \quad$ on $\operatorname{Tor}\left(U\left(A_{k}\right) / \overline{D U\left(A_{k}\right)}\right)$.

By [3, Lemma 10.8] we see that $s_{\kappa}=\Phi$ on $\operatorname{Tor}(U(A) / \overline{D U(A)})$.

To prove uniqueness, let $v \in K L(A, B)$ be another element such that $v_{*}=\varphi_{0}$ on $K_{0}(A), v_{*}=\varphi_{1}$ on $K_{1}(A)$ and $s_{v}=\Phi$ on $\operatorname{Tor}(U(A) / \overline{D U(A)})$. By passing to a subsequence, we may assume that there is an element $v_{k}$ in $K L\left(A_{k}, B_{k}\right)$ such that $\left[\beta_{k, \infty}\right] \cdot v_{k}=v \cdot\left[\alpha_{k, \infty}\right]$. By passing to a subsequence again we may assume that $\psi_{k *}=v_{k *}$ on $K_{0}(A)$ as well as on $K_{1}(A)$. By Theorem 2.6 there exists a unital *-homomorphism $\xi_{k}: A_{k} \rightarrow B_{k}$ such that $\left[\xi_{k}\right]=v_{k}$ in $K L\left(A_{k}, B_{k}\right)$. Then

$$
\beta_{k, \infty}^{\#} \circ \xi_{k}^{\#}=s_{v} \circ \alpha_{k, \infty}^{\#}=s_{\kappa} \circ \alpha_{k, \infty}^{\#}=\beta_{k, \infty}^{\#} \circ \psi_{k}^{\#}
$$

on $\operatorname{Tor}\left(U\left(A_{k}\right) / \overline{D U\left(A_{k}\right)}\right)$. By passing to subsequences again, we may by [3, Lemma 10.4] assume that $\xi_{k}^{\#}=\psi_{k}^{\#}$ on any given finite subset of $\operatorname{Tor}\left(U\left(A_{k}\right) /\right.$ 
$\left.\overline{D U\left(A_{k}\right)}\right)$. Hence, we can arrange that $\xi_{k}{ }^{*}=\psi_{k}{ }^{*}$ on $K^{0}\left(B_{k}\right)$ by Proposition 2.7. It follows from Theorem 2.6 that $\left[\xi_{k}\right]=\left[\psi_{k}\right]$ in $K L\left(A_{k}, B_{k}\right)$. Thus, $\kappa \cdot\left[\alpha_{k, \infty}\right]=v \cdot\left[\alpha_{k, \infty}\right]$ for all $k$. It follows that $\kappa=v$ by [5, Lemma 5.8].

\section{Main results}

In [3] the existence result [3, Theorem 11.2] was subsequently simplified in the case where $K_{0}(A)$ is non-cyclic. The following theorem shows that a similar simplification is possible when $K_{0}(A)$ and $K_{0}(B)$ are cyclic, but this time without $K L$.

THEOREM 4.1. Let $A$ and $B$ be unital simple inductive limits of sequences of finite direct sums of building blocks and assume that $K_{0}(A) \cong \mathrm{Z}, K_{0}(B) \cong \mathrm{Z}$ and that $B$ is infinite dimensional. Let $\varphi_{T}: T(B) \rightarrow T(A)$ be an affine continuous map, let $\varphi_{0}: K_{0}(A) \rightarrow K_{0}(B)$ be an order unit preserving group homomorphism, let $\varphi_{1}: K_{1}(A) \rightarrow K_{1}(B)$ be a group homomorphism, and let $\Phi: U(A) / \overline{D U(A)} \rightarrow U(B) / \overline{D U(B)}$ be a homomorphism such that the diagram

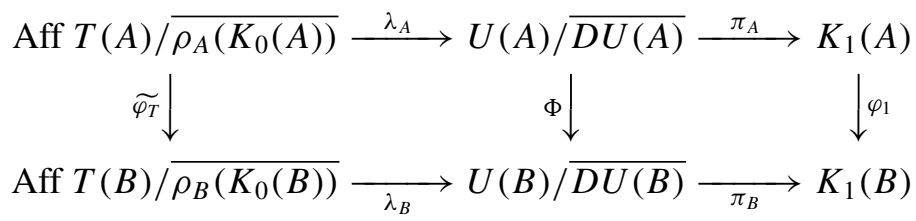

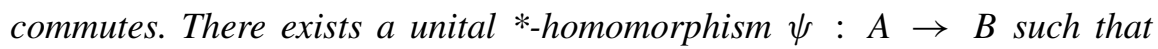
$\psi^{*}=\varphi_{T}$ on $T(B), \psi^{\#}=\Phi$ on $U(A) / \overline{D U(A)}$, and $\psi_{*}=\varphi_{0}$ on $K_{0}(A)$.

Proof. We may assume that $A$ is infinite dimensional. By Theorem 3.3 there exists an element $\kappa \in K L(A, B)$ such that $\kappa_{*}=\varphi_{0}$ on $K_{0}(A), \kappa_{*}=\varphi_{1}$ on $K_{1}(A)$, and $s_{\kappa}=\Phi$ on $\operatorname{Tor}(U(A) / \overline{D U(A)})$. By [3, Theorem 11.2] there exists a unital *-homomorphism $\psi: A \rightarrow B$ such that $[\psi]=\kappa$ in $K L(A, B)$, $\psi^{*}=\varphi_{T}$ on $T(B)$, and $\psi^{\#}=\Phi$ on $U(A) / \overline{D U(A)}$.

The next result says that $K L$ can also be removed from the uniqueness theorem, [3, Theorem 11.5], when $K_{0}(B)$ is cyclic.

THEOREM 4.2. Let $A$ and $B$ be simple unital inductive limit of sequences of finite direct sums of building blocks such that $K_{0}(A) \cong \mathrm{Z}$ and $K_{0}(B) \cong \mathrm{Z}$. Let $\varphi, \psi: A \rightarrow B$ be unital $*$-homomorphisms with $\varphi^{\#}=\psi^{\#}$ on $U(A) / \overline{D U(A)}$. Then $\varphi$ and $\psi$ are approximately unitarily equivalent.

Proof. We may assume that $A$ is infinite dimensional. As in the proof of Theorem 3.3 we see that $A$ is the inductive limit of a sequence

$$
A_{1} \stackrel{\alpha_{1}}{\longrightarrow} A_{2} \stackrel{\alpha_{2}}{\longrightarrow} A_{3} \stackrel{\alpha_{3}}{\longrightarrow} \ldots
$$


of building blocks with unital and injective connecting maps. Similarly $B$ is the inductive limit of a sequence

$$
B_{1} \stackrel{\beta_{1}}{\longrightarrow} B_{2} \stackrel{\beta_{2}}{\longrightarrow} B_{3} \stackrel{\beta_{3}}{\longrightarrow} \ldots
$$

of building blocks with unital and injective connecting maps. By [3, Lemma 8.5] we have that $s\left(B_{k}\right) \rightarrow \infty$. Obviously $\varphi_{*}=\psi_{*}$ on $K_{0}(A)$ and $\varphi_{*}=\psi_{*}$ on $K_{1}(A)$, such that $[\varphi]=[\psi]$ in $K L(A, B)$ by Theorem 3.3. Finally note that $\varphi^{\#}=\psi^{\#}$ implies $\widetilde{\varphi}=\widetilde{\psi}$. Thus the linear map $\widehat{\varphi}-\widehat{\psi}$ takes values in $\overline{\rho_{B}\left(K_{0}(B)\right)}$, and hence it must be 0 . Therefore $\varphi^{*}=\psi^{*}$ on $T(B)$. It follows from [3, Theorem 11.5] that $\varphi$ and $\psi$ are approximately unitarily equivalent.

We need the following isomorphism version of Theorem 4.1.

THEOREM 4.3. Let $A$ and $B$ be simple unital infinite dimensional inductive limits of sequences of finite direct sums of building blocks with $K_{0}(A) \cong$ Z. Let $\varphi_{0}: K_{0}(A) \rightarrow K_{0}(B)$ be an isomorphism of ordered groups with order units, let $\varphi_{1}: K_{1}(A) \rightarrow K_{1}(B)$ be an isomorphism of groups, let $\varphi_{T}$ : $T(B) \rightarrow T(A)$ be an affine homeomorphism, and let $\Phi: U(A) / \overline{D U(A)} \rightarrow$ $U(B) / \overline{D U(B)}$ be an isomorphism of groups, such that the diagram

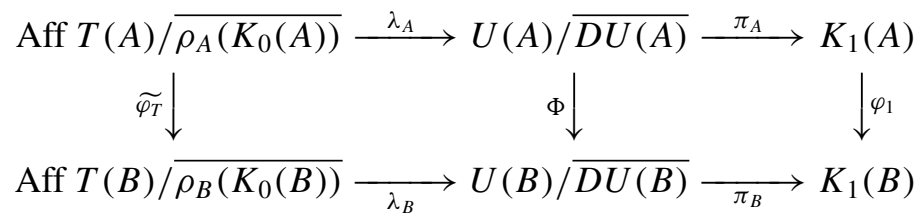

commutes. Then there exists an isomorphism $\psi: A \rightarrow B$ such that $\psi_{*}=\varphi_{1}$ on $K_{1}(A), \psi^{*}=\varphi_{T}$ on $T(B)$, and $\psi^{\#}=\Phi$ on $U(A) / \overline{D U(A)}$.

Proof. By Theorem 4.1 there exists a unital *-homomorphism $\mu: A \rightarrow B$ such that $\mu^{\#}=\Phi$ on $U(A) / \overline{D U(A)}, \mu^{*}=\varphi_{T}$ on $T(B)$, and $\mu_{*}=\varphi_{1}$ on $K_{1}(A)$. Similarly, there exists a unital $*$-homomorphism $\xi: B \rightarrow A$ such that $\xi^{\#}=\Phi^{-1}$ on $U(B) / \overline{D U(B)}, \xi^{*}=\varphi_{T}^{-1}$ on $T(A)$, and $\xi_{*}=\varphi_{1}^{-1}$ on $K_{1}(B)$. By Theorem 4.2 we see that $\mu \circ \xi$ and $\xi \circ \mu$ are approximately inner. Hence by [4, Proposition A] $\mu$ is approximately unitarily equivalent to an isomorphism $\psi: A \rightarrow B$.

We are now in a position to prove part (ii) of Theorem 1.1.

THEOREM 4.4. Let A be a simple unital inductive limit of a sequence of finite direct sums of building blocks with $K_{0}(A) \cong \mathrm{Z}$. Then

$$
\operatorname{Aut}(A) / \overline{\operatorname{Inn}(A)} \cong \operatorname{Hom}\left(K_{1}(A), \operatorname{Aff} T(A) / \overline{\rho_{A}\left(K_{0}(A)\right)}\right) \rtimes \operatorname{Aut}\left(\mathscr{E}_{A}\right),
$$


where the action of $\left(\varphi_{0}, \varphi_{1}, \varphi_{T}\right) \in \operatorname{Aut}\left(\mathscr{E}_{A}\right)$ is given by

$$
\eta \mapsto{\widetilde{\varphi_{T}}}^{-1} \circ \eta \circ \varphi_{1}^{-1}, \quad \eta \in \operatorname{Hom}\left(K_{1}(A), \operatorname{Aff} T(A) / \overline{\rho_{A}\left(K_{0}(A)\right)}\right) .
$$

Proof. We may assume that $A$ is infinite dimensional. By Proposition 2.3 we may identify $U(A) / \overline{D U(A)}$ with $G_{1} \oplus G_{2}$, where $G_{1}=$ Aff $T(A) /$ $\overline{\rho_{A}\left(K_{0}(A)\right)}$ and $G_{2}=K_{1}(A)$. Thus an endomorphism $\psi$ of the group $U(A) /$ $\overline{D U(A)}$ can be identified with a $2 \times 2$ matrix

$$
\left(\begin{array}{ll}
\psi_{11} & \psi_{12} \\
\psi_{21} & \psi_{22}
\end{array}\right)
$$

where $\psi_{i j}: G_{j} \rightarrow G_{i}$ is a homomorphism, $i, j=1$, 2. Note that if $\psi$ is induced by an automorphism of $A$ then $\psi_{21}=0$ since the short exact sequence of Proposition 2.3 is natural.

Let $H=\operatorname{Hom}\left(K_{1}(A)\right.$, Aff $\left.T(A) / \overline{\rho_{A}\left(K_{0}(A)\right)}\right)$. Let $\eta \in H$. Choose by Theorem 4.3 an element $\psi \in \operatorname{Aut}(A)$ such that $\psi^{*}=i d$ on $T(A), \psi_{*}=i d$ on $K_{1}(A)$, and

$$
\psi^{\#}=\left(\begin{array}{cc}
i d & \eta \\
0 & i d
\end{array}\right)
$$

on $U(A) / \overline{D U(A)}$. By Theorem 4.2 we obtain a well-defined group homomorphism

$$
\iota: H \rightarrow \operatorname{Aut}(A) / \overline{\operatorname{Inn}(A)}
$$

by setting $\iota(\eta)=p(\psi)$, where $p: \operatorname{Aut}(A) \rightarrow \operatorname{Aut}(A) / \overline{\operatorname{Inn}(A)}$ denotes the canonical map. Let $\pi: \operatorname{Aut}(A) / \overline{\operatorname{Inn}(A)} \rightarrow \operatorname{Aut}\left(\mathscr{E}_{A}\right)$ be the homomorphism

$$
\pi(p(\psi))=\left(\psi_{*}, \psi_{*},\left(\psi^{*}\right)^{-1}\right) .
$$

We have a short exact sequence

$$
0 \longrightarrow H \stackrel{\iota}{\longrightarrow} \operatorname{Aut}(A) / \overline{\operatorname{Inn}(A)} \stackrel{\pi}{\longrightarrow} \operatorname{Aut}\left(\mathscr{E}_{A}\right) \longrightarrow 0
$$

of groups. Let $\left(\varphi_{0}, \varphi_{1}, \varphi_{T}\right) \in \operatorname{Aut}\left(\mathscr{E}_{A}\right)$. Choose by Theorem 4.1 an element $\psi$ in $\operatorname{Aut}(A)$ such that $\psi_{*}=\varphi_{1}, \psi^{*}=\varphi_{T}^{-1}$, and

$$
\psi^{\#}=\left(\begin{array}{cc}
{\widetilde{\varphi_{T}}}^{-1} & 0 \\
0 & \varphi_{1}
\end{array}\right) \text {. }
$$

By Theorem 4.2 we obtain a well-defined map $\sigma: \operatorname{Aut}\left(\mathscr{E}_{A}\right) \rightarrow \operatorname{Aut}(A) / \overline{\operatorname{Inn}(A)}$ by setting $\sigma\left(\varphi_{0}, \varphi_{1}, \varphi_{T}\right)=p(\psi)$. Note that $\sigma$ splits the sequence above. Hence $\operatorname{Aut}(A) / \overline{\operatorname{Inn}(A)}$ is isomorphic to a semi-direct product $H \rtimes \operatorname{Aut}\left(\mathscr{E}_{A}\right)$. Since

$$
\iota\left({\widetilde{\varphi_{T}}}^{-1} \eta \varphi_{1}^{-1}\right)=\sigma\left(\varphi_{0}, \varphi_{1}, \varphi_{T}\right) \iota(\eta) \sigma\left(\varphi_{0}, \varphi_{1}, \varphi_{T}\right)^{-1},
$$


it follows that the action of $\operatorname{Aut}\left(\mathscr{E}_{A}\right)$ on $H$ is the desired one.

Let us finally show that our main result can be simplified when $K_{1}(A)$ is a torsion group. Recall that $\operatorname{ext}(G, H)$ is defined as $\operatorname{Ext}(G, H) / \operatorname{Pext}(G, H)$ for abelian groups $G$ and $H$, where $\operatorname{Pext}(G, H)$ is the subgroup of pure (i.e. locally trivial) extensions in $\operatorname{Ext}(G, H)$, see [5, Chapter 5].

Corollary 4.5. Let A be a simple unital inductive limit of a sequence of finite direct sums of building blocks such that $K_{1}(A)$ is a torsion group. Then

$$
\left.\operatorname{Aut}(A) / \overline{\operatorname{Inn}(A)} \cong \operatorname{ext}\left(K_{1}(A), K_{0}(A)\right)\right) \rtimes \operatorname{Aut}\left(\mathscr{E}_{A}\right),
$$

where the action of $\left(\varphi_{0}, \varphi_{1}, \varphi_{T}\right) \in \operatorname{Aut}\left(\mathscr{E}_{A}\right)$ is given by $e \mapsto \varphi_{0 *} \circ \varphi_{1}^{-1 *}(e)$ for $e \in \operatorname{ext}\left(K_{1}(A), K_{0}(A)\right)$.

Proof. If $K_{0}(A)$ is non-cyclic, then Aff $T(A) / \overline{\rho_{A}\left(K_{0}(A)\right)}$ is torsion-free by [3, Lemma 10.3], and hence the result follows in this case from (i) in Theorem 1.1. Therefore we may assume that $K_{0}(A) \cong Z$. Then $\rho_{A}$ is injective and has closed range, and hence we have a short exact sequence

$$
0 \longrightarrow K_{0}(A) \stackrel{\rho_{A}}{\longrightarrow} \text { Aff } T(A) \stackrel{q_{A}}{\longrightarrow} \text { Aff } T(A) / \overline{\rho_{A}\left(K_{0}(A)\right)} \longrightarrow 0 .
$$

Let $E$ denote the corresponding class in $\operatorname{Ext}\left(\operatorname{Aff} T(A) / \overline{\rho_{A}\left(K_{0}(A)\right)}, K_{0}(A)\right)$. Note that $\operatorname{Aff} T(A)$ is divisible, and therefore $\operatorname{Ext}\left(K_{1}(A)\right.$, Aff $\left.T(A)\right)=0$. Hence by applying [2, Theorem III.3.4] we get an isomorphism

$$
E_{*}: \operatorname{Hom}\left(K_{1}(A), \operatorname{Aff} T(A) / \overline{\rho_{A}\left(K_{0}(A)\right)}\right) \rightarrow \operatorname{Ext}\left(K_{1}(A), K_{0}(A)\right),
$$

where $E_{*}(\eta)=\eta^{*}(E)$. By a result of C. U. Jensen, see e.g. [8, Theorem 6.1], we have that $\operatorname{Pext}\left(K_{1}(A), K_{0}(A)\right)=0$. Thus $\operatorname{Ext}\left(K_{1}(A), K_{0}(A)\right)=\operatorname{ext}\left(K_{1}(A)\right.$, $\left.K_{0}(A)\right)$. To see that the two actions of $\operatorname{Aut}\left(\mathscr{E}_{A}\right)$ can be identified as well, note that the diagram

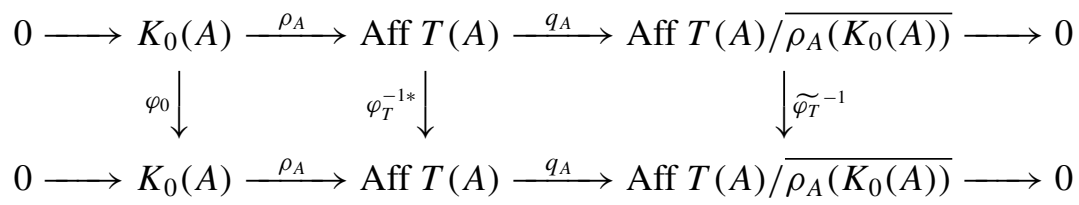

commutes, such that ${\widetilde{\varphi_{T}}}^{-1 *}(E)=\varphi_{0_{*}}(E)$ by [2, Proposition III.1.8]. The corollary follows.

We mention without proof that the $C^{*}$-algebras considered in the above corollary are exactly the simple unital inductive limits of sequences of finite direct sums of building blocks of the form

$$
\left\{f \in C[0,1] \otimes M_{n}: f\left(x_{i}\right) \in M_{d_{i}}, i=1,2, \ldots, N\right\} .
$$


Corollary 4.5 suggests that only $K L$, and not $U(\cdot) / \overline{D U(\cdot)}$, is needed in an approximate intertwining argument to show that the Elliott invariant is a classifying invariant for these $C^{*}$-algebras. This was demonstrated by Jiang and $\mathrm{Su}$ [1] for a large subclass of this class of $C^{*}$-algebras.

Let us finally emphasize the following surprising consequence of the corollary above. Let $A$ be a simple unital inductive limit of a sequence of finite direct sums of building blocks. If $K_{0}(A)$ is non-cyclic then $\operatorname{Aut}(A) / \overline{\operatorname{Inn}(A)}$ is isomorphic to a semi-direct product

$$
\left(\operatorname{Hom}\left(K_{1}(A), \operatorname{Aff} T(A) / \overline{\rho_{A}\left(K_{0}(A)\right)}\right) \times \operatorname{ext}\left(K_{1}(A), K_{0}(A)\right)\right) \rtimes \operatorname{Aut}\left(\mathscr{E}_{A}\right) .
$$

The term $\operatorname{Hom}\left(K_{1}(A)\right.$, Aff $\left.T(A) / \overline{\rho_{A}\left(K_{0}(A)\right)}\right)$ vanishes e.g. if $A$ has real rank zero, whereas the term $\operatorname{ext}\left(K_{1}(A), K_{0}(A)\right)$ vanishes e.g. if $A$ is an inductive limit of a sequence of finite direct sums of circle algebras. When $K_{0}(A) \cong \mathrm{Z}$ and $K_{1}(A)$ is a torsion group, however, these two terms agree, but only one of them appear in the expression for $\operatorname{Aut}(A) / \overline{\operatorname{Inn}(A)}$.

\section{REFERENCES}

1. Jiang, X., and $\mathrm{Su}, \mathrm{H}$. , On a simple unital projectionless $C^{*}$-algebra, Amer. J. Math. 121 (1999), 359-413.

2. Mac Lane, S., Homology, Springer Verlag, 1963.

3. Mygind, J., Classification of certain simple $C^{*}$-algebras with torsion in $K_{1}$, Canad. J. Math. 53 (2001), 1223-1308.

4. Rørdam, M., A short proof of Elliott's theorem: $\mathscr{O}_{2} \otimes \mathscr{O}_{2} \cong \mathscr{O}_{2}$, C. R. Math. Rep. Acad. Sci. Canada 16 (1994), 31-36.

5. Rørdam, M., Classification of certain infinite simple $C^{*}$-algebras, J. Funct. Anal. 131 (1995), $415-458$.

6. Rosenberg, J., and Schochet, C., The Künneth theorem and the universal coefficient theorem for Kasparov's generalized K-functor, Duke Math. J. 55 (1987), 431-474.

7. Schochet, C., Topological methods for $C^{*}$-algebras. III. Axiomatic homology, Pacific J. Math. 114 (1984), 399-445.

8. Schochet, C., A Pext primer: Pure extensions and $\lim ^{1}$ for infinite abelian groups, New York J. Math. Monographs 1 (2003).

9. Thomsen, K., Limits of certain subhomogeneous $C^{*}$-algebras, Mém. Soc. Math. Fr. 71 (1997).

INSTITUT FOR MATEMATISKE FAG

AARHUS UNIVERSITET

BYGNING 530

8000 ÅRHUS C

DENMARK
CURRENT ADDRESS: SKOVKILDEPARKEN 139 8660 SKANDERBORG DENMARK

E-mail: jmygind@hotmail.com 